\title{
ELUCIDATION OF TYPE OF MOLECULAR INTERACTIONS FOR THE IMPRINTING OF SHIKIMIC ACID
}

\author{
YEOH CHEE BENG*; NOR AZAH YUSOF**; CHONG CHIEW LET*; \\ SAW MEI HUEY* and TAN YEW Al*
}

\begin{abstract}
During the preparation of shikimic acid imprinted polymer, the effect of rebinding solution water content on the rebinding performance of shikimic acid (SA) imprinted polymer was studied. Result showed that the highest affinity toward SA was in a 100\% aqueous rebinding solution, suggesting that the interactions occurred between $S A$ and the polymer were ionic and/or hydrophobic in nature. Further study on the effect of rebinding solution $\mathrm{pH}$ to the $S A$ imprinted polymer rebinding performance showed that the performance of the SA imprinted polymer depended on the rebinding solution $\mathrm{pH}$ and the highest affinity occurred at $\mathrm{pH}$ 4.0; this confirmed that ionic interaction played an important role in the rebinding of SA. Taking into account the deductions made from the combined results of the rebinding solution water content and effect of $\mathrm{pH}$, together with observation of the red shift in the SA spectral during ultraviolet-visible spectroscopic titration experiments, suggest that the specificity shown by SA imprinted polymer in the aqueous solution was due to both ionic and $\pi-\pi$ hydrophobic interactions.
\end{abstract}

\section{Keywords: molecular imprinted polymer, shikimic acid, ultraviolet-visible spectroscopic titration.}

Date received: 1 March 2016; Sent for revision: 21 March 2016; Received in final form: 27 July 2016; Accepted: 28 July 2016.

\section{INTRODUCTION}

Molecular imprinted polymer are highly crosslinked polymeric materials that are formed during the polymerisation process in the presence of template molecules. During polymerisation, template molecules and functional monomers undergo self-assembly process as a result of bonding interactions such as covalent bonding, hydrogen bonding, van der Waals interaction, $\pi-\pi$ interaction

\footnotetext{
Malaysian Palm Oil Board,

6 Persiaran Institusi, Bandar Baru Bangi,

43000 Kajang, Selangor,

Malaysia.

E-mail: cbyeoh@mpob.gov.my

** Department of Chemistry,

Faculty of Science,

Universiti Putra Malaysia,

43400 UPM Serdang, Selangor, Malaysia.
}

and others. Output from the self-assembly process is the formation of a polymer with the functional monomers framing the template molecules. Upon removal of the template molecules, the polymer now has voids in them in the shape of the template molecules. These voids are the sites which are capable of rebinding with the specific template or its analogue.

It is well-known that the poor ability of imprinted polymer to bind selectively in the aqueous phase is one of the issues that need to be addressed in the field of molecular imprinted polymer preparation. Unfortunately, many target molecules of interest are only present in aqueous media such as body fluids, wastewater and river. Shikimic acid (SA) is a compound that has a very high solubility in water $\left(18 \mathrm{~g} \bullet\right.$ litre $\left.^{-1}\right)$, due to its high medicinal value, the research in the area of SA 
production and purification has become one of the hottest topics in the respective fields.

Previous preliminary research (Yeoh et al., 2015) has shown that SA imprinted polymer prepared using 4-Vinylpyrine (4VP) and ethylene glycol dimethacrylate (EDGMA) as the functional monomer and cross-linker respectively with a template: monomer: cross-linker mol ratio of 1:6:10 in $10 \mathrm{ml}$ methanol/water $(4: 1, \mathrm{v} / \mathrm{v})$, was selective toward SA in aqueous environment. However, no details on the optimisation of rebinding conditions such as water content and $\mathrm{pH}$ of the rebinding solution that led to the optimum rebinding performance as well as the explanations to the types of interactions responsible for the selectivity of SA imprinted polymer were reported.

The effects of water content and $\mathrm{pH}$ of the rebinding solutions on the performance of the SA imprinted polymer is the subject of this article. These effects, together with the results of studies on the template-functional monomer interactions using ultraviolet-visible (UV-Vis) spectroscopic titration method are used to deduce the possible interaction between SA and 4VP.

\section{MATERIALS AND METHODS}

\section{Chemicals and Reagents}

SA [98\% purity by high performance liquid chromatography (HPLC)] was obtained from Hangzhou API Chem Technology Co. Ltd, China. The 4VP (95\% purity), EGDMA, (98\% purity) and 4,4-Azobis(4-cyanovaleric acid) (ACVA, $\geq 75 \%$ purity) were from Sigma Aldrich. All solvents used were at least A.R. grade from Fisher Chemicals. Spectroscopic grade methanol from Sigma Aldrich was used in UV-Vis spectroscopic titration experiment and Mili Q ultra-pure water with 18.2 $\mathrm{M} \Omega \bullet \mathrm{cm}^{-1}$ resistivity was used throughout the study.

\section{Preparation of Shikimic Acid Imprinted Polymer}

Steps in SA imprinted polymer preparation involved the mixing of $1 \mathrm{mmol}$ of SA (template) and $6 \mathrm{mmol}$ of $4 \mathrm{VP}$ in $10.0 \mathrm{ml}$ of methanol-water $(4: 1, \mathrm{v} / \mathrm{v})$ (Haupt et al., 1998) as a porogen. After $12 \mathrm{hr}$ standing in the refrigerator under dark condition at a temperature of $2^{\circ} \mathrm{C}-6^{\circ} \mathrm{C}, 10 \mathrm{mmol}$ of EGDMA was added together with $22.0 \mathrm{mg}$ of radical initiator, ACVA. The mixture was then sonicated for $10 \mathrm{~min}$ under gentle nitrogen gas bubbling. Then, polymerisation was carried out by placing the mixture in water bath at $60^{\circ} \mathrm{C}$ for $24 \mathrm{hr}$. Control polymer was prepared following the same procedures except no template was added in the preparation.
After the polymerisation, the glass container was cracked and the polymer obtained was ground in a short and repeated cycles (approximately 5 min per cycle) using mortar and pestle. Between each grinding cycle, the product was dry sieved to obtain polymer with a size of approximately $125 \mu \mathrm{m}$. The fine polymer particles collected were then subjected to Soxhlet extraction using 10\% acetic acid in methanol solution for a period of $24 \mathrm{hr}$ followed by extraction with methanol for another $24 \mathrm{hr}$ to remove the template and wash off any residual acetic acid in the polymer. The polymer particles were then subjected to three times $220 \mathrm{ml}$ acetone sedimentation process in a $250 \mathrm{ml}$ measuring cylinder to remove fine particles that were not suitable for subsequent studies. Particles that remained floating after 15 min standing were discarded and the rest were then vacuum dried at $40^{\circ} \mathrm{C}$ for $24 \mathrm{hr}$ and the final dried polymer particles were now ready for rebinding evaluations.

\section{General Procedures for Batch Rebinding Experiment}

Rebinding experiments for SA imprinted polymer and its corresponding control were conducted by allowing $20 \mathrm{mg}$ of the processed polymer particles to mix well with $1.5 \mathrm{ml}$ of 100 $\mu \mathrm{g} \bullet \mathrm{ml}^{-1}$ of SA solution (prepared in various solvents as described) in a $2 \mathrm{ml}$ Eppendorf tube. The mixture was then shaken on the shaker for a period of $12 \mathrm{hr}$ followed by centrifuging at $3000 \mathrm{rpm}$ for $5 \mathrm{~min}$ to enable phase separation. The top layer was taken for HPLC analysis to determine the SA concentration.

Calculations for the $\%$ of rebinding were done using Equation (1):

$$
\begin{aligned}
& \text { [(Amount of SA before adsorption) }- \\
& \frac{\text { (Amount of SA after adsorption }]}{\text { [(Amount of SA before adsorption) }} \times 100 \% \\
& \text { Equation (1) - Calculation of the } \% \text { of rebinding }
\end{aligned}
$$

\section{Effect of Water Content in the Rebinding Solutions}

Rebinding performance of SA imprinted polymer in the solution with different water contents was assessed according to the general procedures described for batch rebinding experiment, using a series of SA solutions prepared in a methanol and water mixed solvent with water content ranging from $0 \%$ to $100 \%(\mathrm{v} / \mathrm{v})$.

\section{Study on the Effect of $\mathrm{pH}$}

To study the effect of $\mathrm{pH}$ on the rebinding performance of the polymer, rebinding experiment was conducted according to the procedures outlined 
for batch rebinding experiment using rebinding

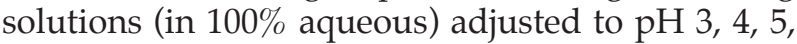
6 and 8 using diluted hydrochloric acid or sodium hydroxide.

\section{High Performance Liquid Chromatography Analysis of Shikimic Acid}

Determination of SA concentration was carried out using an Agilent 1100 series HPLC with UV detection at $213 \mathrm{~nm}$. The column used was Rezex ${ }^{\mathrm{TM}}$ ROA-Organic Acid $\mathrm{H}^{+}(8 \%), 150 \times 7.8 \mathrm{~mm}$ from Phenomenex Inc. operated at room temperature. The mobile phase used was a $0.0025 \mathrm{M}$ sulphuric acid solution at a flow rate of $0.6 \mathrm{ml} \bullet \mathrm{min}^{-1}$ with total run time of $25 \mathrm{~min}$. The concentration of SA was determined by mean of area under the peak.

\section{Ultraviolet-visible Spectroscopic Titration}

For the UV titration experiment, a series of template and functional monomer mixtures were prepared in the ratios of 1:0,1:1, 1:2, 1:4, 1:6, 1:8 and 1:10 in methanol and water $(4: 1, \mathrm{v} / \mathrm{v})$ mixed solvent. The prepared solutions were then mixed well using a vortex mixer for $10 \mathrm{~s}$, before being kept in a refrigerator under dark condition for a period of $12 \mathrm{hr}$ to allow complete reaction of template and functional monomer. Reference solutions made up of the corresponding functional monomer ratios were also prepared and treated in the same manners.

Upon storage, UV-Vis spectrum of the respective template and functional monomer mixture was recorded at room temperature with the corresponding functional monomer solution acting as a reference.

\section{RESULTS AND DISCUSSION}

\section{Effect of Water Content in the Rebinding Solution}

There is no doubt that the presence of water is detrimental to the performance of imprinted polymer that interacts mainly through hydrogen bonding. However, the actions of water molecules in the rebinding solution are random in nature, water molecules interact non-selectively with both the specific binding sites and non-specific one. A report (Zhu et al., 2009) has shown that the additional of a small amount of water in the rebinding solution can drastically improve the performance of an imprinted polymer. This is due to the destruction of non-selective hydrogen bonding binding sites of the polymer and the promotion of hydrophobic and ionic interactions that may co-exist between the template molecule and the functional monomer (Nicholls et al., 1995).

Figure 1 shows the rebinding results for the SA imprinted polymer conducted in the mixed solvent of methanol and water at different proportions. The concentration of SA was $100 \mu \mathrm{g} \bullet \mathrm{ml}^{-1}$. Despite the confusing rebinding performances shown by the polymer in the solutions with $0 \%$ and $10 \%$ water contents, there is an observable trend of increasing absorption of SA by the polymer, both in the SA imprinted polymer and control when the content of water is increased.

This upward trend may be due to the reason that an increase in the water content has changed the overall environment for the rebinding process. As the amount of water is increased, the environment becomes more favourable for the formation of shikimate ions (acid dissociation),

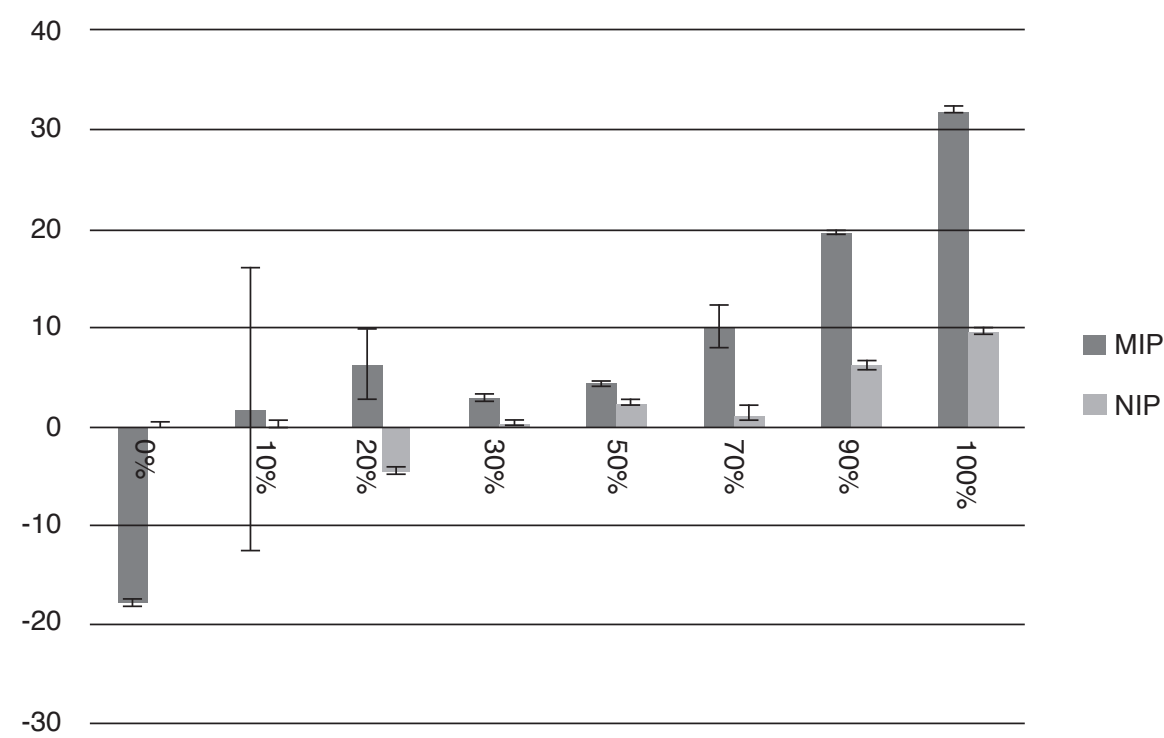

Note: MIP - imprinted polymer. NIP - control.

Figure 1. Rebinding performance of shikimic acid (SA) imprinted polymer in mixed MeOH and water solution with different water contents. Error bars represent 1 standard deviations from rebinding experiments conducted in triplicates. 
resulting in the increase of ionic interaction between the polymer binding sites (the pyridine moiety) and the shikimate ions. The fact that increasing of aqueous composition in an aqueous-organic mixed rebinding solution will promotes the dissociation of SA is supported by the results of several studies (Eckert et al., 2009; Kutt et al., 2006; Kaljurand et al., 2003) on the determination of pKa values of various organic acids in non-aqueous or mixed aqueous and organic solutions. Results from these studies have shown that the $\mathrm{pKa}$ of the organic acids reported was increased with the increasing in the composition of organic solvent (causing less acid dissociation). From the study on the effect of water, it is deduced that ionic interaction is responsible for the binding between SA imprinted polymer and SA.

\section{Effect of Solution $\mathrm{pH}$ on the Performance of Shikimic Acid Imprinted Polymers}

In non-covalent imprinting, the performance of the imprinted polymer can be strongly affected by the $\mathrm{pH}$ of the rebinding solution. This is especially in the carboxyl group of SA has a pKa value of 3.9 (Ding, 2012), at $\mathrm{pH} \cong \mathrm{SA} \mathrm{pKa}$, half of the acid would be dissociated (Figure 3, top). On the other hand, $4 \mathrm{VP}$, expressed in its conjugate acid dissociation form, has a $\mathrm{pKa}$ value of 5.6 (Perrin, 1965) (Figure 3 ). At $\mathrm{pH} 4.0$, which was $\approx 1.6$ unit to the left from its $\mathrm{pKa}$, the majority of $4 \mathrm{VP}(>90 \%)$ existed in its protonated (conjugated acid) form (Figure 3, bottom). Under this condition, the dissociated SA molecules were preferably bound with the positively charged binding sites of the MIP.

At $\mathrm{pH} 6.0$ or more, almost all the SA was dissociated and most of the 4VP was in its neutral form. Shikimate ions were negatively charged, when they approached 4VP molecules, a repulsive force would be formed between the $\pi$ electron clouds at the aromatic ring and the shikimate ions, causing a drastic drop in adsorption as the $\mathrm{pH}$ is increased. Likewise, at lower $\mathrm{pH}$, the majority of SA is in its neutral form. Whereas, almost all of the $4 \mathrm{VP}$ is protonated, the electron deficiency $4 \mathrm{VP}$ will interact marginally with the electron rich portion of SA, causing the slightly better performance of MIP

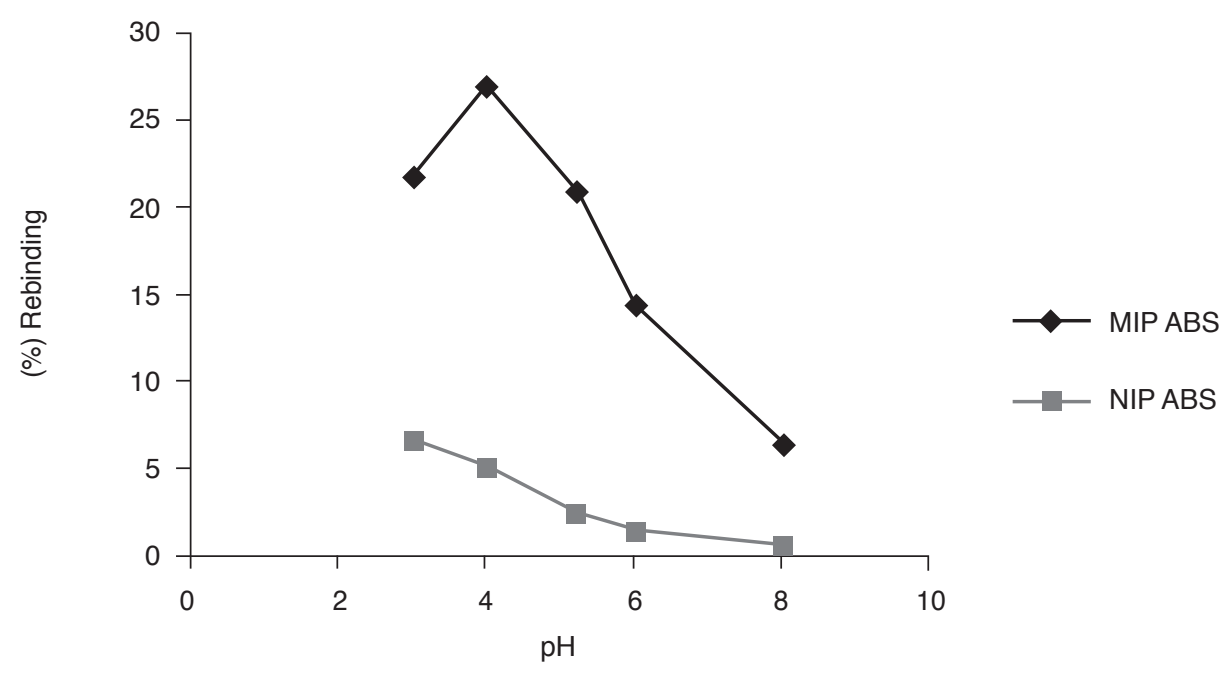

Figure 2. Rebinding performance of shikimic acid (SA) imprinted polymer and its control at different $\mathrm{pH}$.

true if the interactions responsible for polymer selectivity involve the hydrogen atom, irrespective of hydrogen bonding or ionic interaction.

Figure 2 shows the rebinding performance of SA imprinted polymers and control polymers in SA solutions with different $\mathrm{pH}$ from 3.0, 4.0, 5.2, 6.0 and 8.0. It can be seen that maximum adsorption occurred at pH 4.0 (27.2\%) for imprinted polymer with the adsorption rate declining after this $\mathrm{pH}$. Rebinding performance of the control polymer, on the other hand, followed a downward trend without any drastic change.

The appearance of a maximum adsorption for MIP at $\mathrm{pH} 4.0$ is understandable. Hydrogen atom

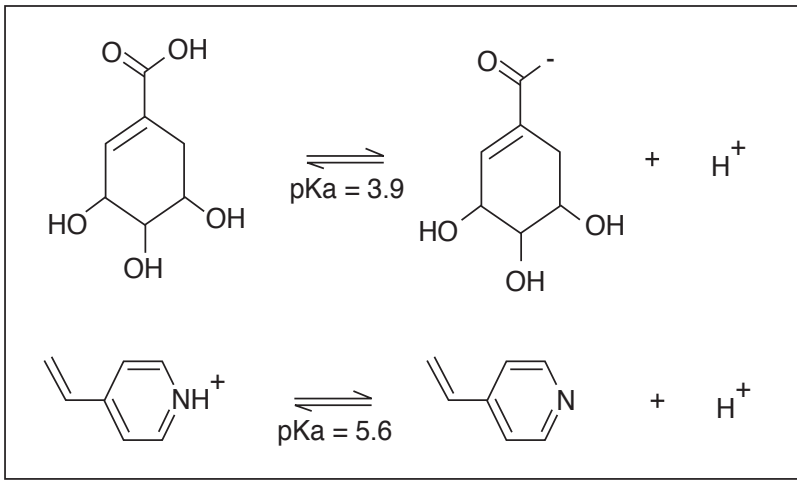

Figure 3. Dissociation of shikimic acid (SA) (top) and 4-Vinylpyrine $(4 V P)$ conjugate acid (bottom). 
under $\mathrm{pH} 3.0$ as compared to the environment when the $\mathrm{pH}$ is more than 6.0.

The results from these $\mathrm{pH}$ effect experiments suggested a strong ionic interaction influence as deduced early. The performance of prepared MIP can largely be controlled via alteration of $\mathrm{pH}$ values of the rebinding solutions.

\section{Ultraviolet-visible Spectroscopic Titration}

The formulation that is able to produce the most stable pre-polymerisation template-monomer complex is always said to have high possibility in producing good imprinted polymer with good rebinding performance. Stability of a prepolymerisation complex can be examined via the study of the strength of the template-monomer interaction in the pre-polymerisation solution. In this study, UV-Vis spectroscopic titration technique was used to determine the best template:monomer
(TM) ratio that shows the strongest interaction in the SA imprinted polymer preparation.

Figure 4 shows the UV spectra of SA-4VP mixtures at various TM ratios ranging from 1:0 to $1: 10$. It can be seen from the figure that as the ratio of $4 \mathrm{VP}$ is increased, a red shift in the $\lambda$ max of the complex formed was observed. The shift in $\lambda$ max is about $8 \mathrm{~nm}$. The red shift of the $\lambda$ max can be explained as a result of the interaction between SA and 4VP (Figure 5). In the solution, SA is partially dissociated into a shikimate ion with the release of proton from its carboxylic acid moiety. The $\pi$ electrons of the carboxylate ion formed are delocalised by resonance, hence, stabilising the ion. Upon the addition of $4 \mathrm{VP}$, the amount of acid dissociation is increased causing the increase in the amount of shikimate ion and the interaction between the aromatic $\pi$ electrons of $4 \mathrm{VP}$ and the $\pi$ electrons of shikimate ion causing the red shift of the maximum absorption wavelength.

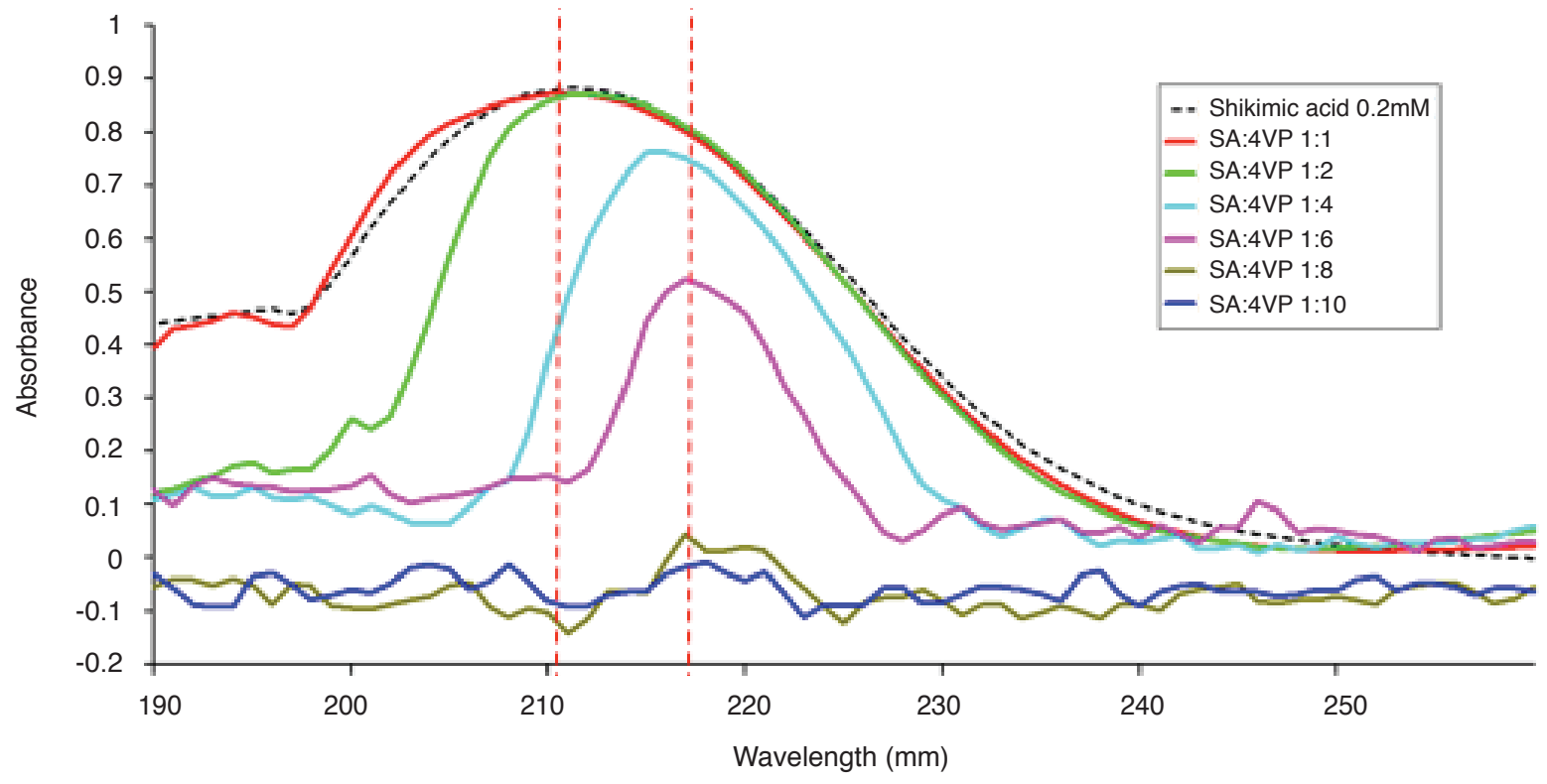

Figure 4. Ultraviolet spectra of shikimic acid (SA) with different molar ratios of SA to 4-Vinylypyrine (4VP).

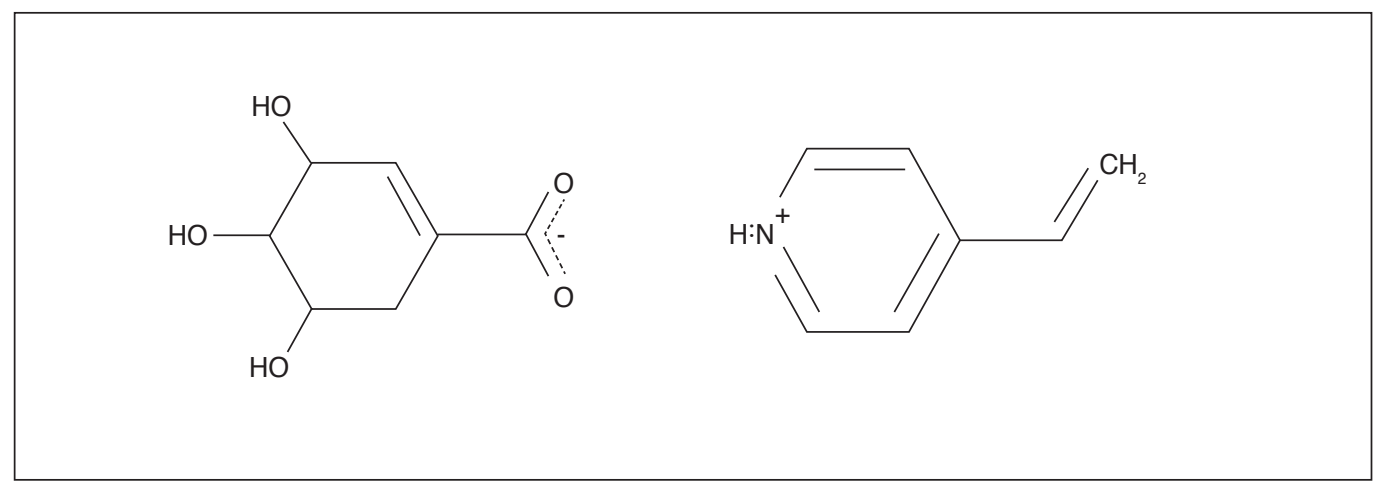

Figure 5. Possible interaction between shikimic acid (SA) and 4-Vinylypyrine (4VP). 


\section{CONCLUSION}

Understanding the type of interaction responsible for the imprinting effect shown by an imprinted polymer is important for the design and improvement of the imprinted polymer. In the course of SA imprinted polymer preparation, it was noted that the polymer performed best in 100\% aqueous solution at $\mathrm{pH}$ 4.0. Based on knowledge of SA dissociation behaviour in an aqueous environment and an understanding of acid-base reaction, it was deduced that the interaction between SA and 4VP is ionic in nature. However, ionic interaction alone is not sufficient to account for the specificity shown by the SA imprinted polymers. Taking the results of the three studies into account, it can be concluded that the specificity shown by SA imprinted polymer are the results of both ionic and $\pi-\pi$ hydrophobic interactions.

\section{REFERENCES}

DING, Z Y (2012). Novel Method for Extracting and Separating Shikimic Acid in Illicium Verum. China patent application CN 201110167965.

ECKERT, F; LEITO, I; KALJURAND, I; KÜTT, A; KLAMT, A and DIEDENHOFEN, M (2009). Prediction of acidity in acetonitrile solution with cosmo-rs. J. Comput. Chem., 30 (5): 799-810.

HAUPT, K; DZGOEV, A and MOSBACH, K (1998). Assay system for the herbicide 2,4-dichlorophenoxyacetic acid using a molecularly imprinted polymer as an artificial recognition element. Anal. Chem., 70 (3): 628-63.

KALJURAND, I; RODIMA, T; PIHL, A; MÄEMETS, V; LEITO, I; KOPPEL, I A and MISHIMA, M (2003). Acid-base equilibria in nonpolar media. 4. Extension of the self-consistent basicity scale in the medium gas-phase basicities of phosphazenes. J. Org. Chem., 68 (26): 9988-9993.

KÜTT, A; LEITO, I; KALJURAND, I; SOOVÄLI, L; VLASOV, V M; YAGUPOLSKII, L M and KOPPEL, I A (2006). A comprehensive self-consistent spectrophotometric acidity scale of neutral brønsted acids in acetonitrile. J. Org. Chem., 71 (7): 2829-2838.

NICHOLLS, I A; RAMSTRÖM, O and MOSBACH, K (1995). Insights into the role of the hydrogen bond and hydrophobic effect on recognition in molecularly imprinted polymer synthetic peptide receptor mimics. J. Chromatogr. A, 691 (1-2): 349-353.

PERRIN, D D (1965). IUPAC Chemical Data Series: Dissociation Constants of Organic Bases in Aqueous Solution. Butterworths: London.

YEOH, C B; NORAZAH, Y; CHONG, C L; SAW, M H and TAN, Y A (2015). Study toward the preparation of aqueous compatible shikimic acid imprinted polymer. J. Oil Palm Res. Vol. 27 (1): 90-96.

ZHU, X F; CAO, Q E; YANG, X Q; LI, F; WANG, G S and DING, Z T (2009). Preparation and recognition mechanism of gallic acid imprinted polymers. Helv. Chim. Acta, 92 (1): 78-87. 